\title{
Effects of supplementing freeze-dried Mitsuokella jalaludinii phytase on the growth performance and gut microbial diversity of broiler chickens
}

\begin{abstract}
Inclusion of phytase in animal feedstuff is a common practice to enhance nutrients availability. However, little is known about the effects of phytase supplementation on the microbial ecology of the gastrointestinal tract. In this study, freeze-dried Mitsuokella jalaludinii phytase (MJ) was evaluated in a feeding trial with broilers fed a low available phosphorus (aP) diet. A total of 180 male broiler chicks (day-old Cobb) were assigned into three dietary treatments: Control fed with $0.4 \%$ (w/w) of available phosphorus (aP); Group T1 fed low aP [0.2\% (w/w)] supplemented with MJ; and T2 fed low aP and deactivated MJ. The source of readily available $\mathrm{P}$, dicalcium phosphate (DCP), was removed from low aP diet, whereby additional limestone was provided to replace the amount of Ca normally found in DCP. For each treatment, 4 replicate pens were used, where each pen consisted of 15 animals. The animals' energy intake and caecal bacterial community were monitored weekly for up to 3 weeks. The apparent metabolizable energy (AME) and apparent digestibility of dry matter (ADDM) of broilers fed with different diets were determined. In addition, the caecal microbial diversities of broilers were assessed using high-throughput next-generation sequencing targeting the V3-V4 region of bacterial 16S rRNA. The results showed that broilers fed with T1 diet have better feed conversion ratio $(F C R)$ when compared to the Control $(p<.05)$ and T2 diets $(p<.05)$, demonstrating the efficiency of MJ as a supplement to low aP diet. Nevertheless, MJ did not significantly affect the microbial population and diversity in broilers' caeca, which mainly consists of members from Bacteroidetes, Firmicutes, and Proteobacteria. Regardless, significant variations in the caecal bacterial composition were observed over time, probably due to succession as the broilers aged. This is the first reported study on the effect of MJ on the microbial diversity of broiler's caeca.
\end{abstract}

Keyword: Mitsuokella jalaludinii; Bacterial phytase; Feed supplement; Gut microbial population 